\title{
MOLECULAR GENETIC ANALYSIS THREE TYPES OF CYTOPLASMIC MALE STERILITY IN MAIZE
}

\author{
Prysiazhniuk Larysa ${ }^{l}$, Otroshko Snizhana ${ }^{l}$, Honcharov Yu. ${ }^{2}$, Melnyk $S .{ }^{1}$, Tahantsova Maryna ${ }^{l}$ \\ ${ }^{l}$ Ukrainian Institute for Plant Variety Examination, ${ }^{2}$ Research Institute of Agrarian Business \\ e-mail: prysiazhniuk_1@ukr.net
}

The commercial production of hybrid seed involves the using of cytoplasmic male sterility (CMS). There are several types of CMS associated with different mitochondrial regions and Rf (restorer of fertility) genes. The most common are T-type CMS (Texas), C-type (Charrua), and Stype (USDA). The using of T-type has been reducing since 1970s because it confers susceptibility to Helminthosporium maydis. It is known, that test crossing is the most common method for investigation maize cytoplasm. This approach requires a lot of field work and time. Knowledge about the molecular structures and the mechanisms underlying CMS increased considerably with the development of molecular approaches. The method of polymerase chain reaction (PCR) developed for this purpose is reliable and faster. The aim of our research was to study of the molecular genetic polymorphism of mitochondrial regions associated with CSF in maize of Ukrainian origin.

Thirty four inbred lines of maize developed in Ukraine by Research Institute of Agrarian Business were used. Inbred lines were divided into groups according to types of sterility. There were five inbred lines with S-type of cytoplasm, five with C-type and four - probably T-type. Also, five pairs of lines with normal cytoplasm and their sterile analogs of S-type and five pairs of C-type were investigated. All samples of $\mathrm{S}$ - and C-types of CMS were taken in the field according to morphological characteristics; the samples of T-type were got from collection of this source. DNA isolation was extracted from $100 \mathrm{mg}$ of leafs and 5-day maize sprouts using CTAB and dissolving DNA in TE buffer. PCR reactions were performed by Liu et al. (2002). To visualize the amplification products, electrophoresis was performed in $2 \%$ agarose gel.

All five lines of maize of S-type CMS have shown the bands of an expected size $799 \mathrm{bp}$. The sterility of the inbred lines, which are sterile analogs with S-type of cytoplasm, was confirmed. However, the one line, which was announced as line with normal cytoplasm, proved the sterile one. It is proved with the presence the allele of $799 \mathrm{bp}$. Considering the fact that leafs were obtained from growing plant, it can be explained that the plants of maize have the anthers, but they were not to be able to disperse the pollen. According to obtained results, the inbred lines, which had a normal cytoplasm, were characterized of presence from two to three alleles. The sizes of these alleles were 632, 855, 1088 and $1145 \mathrm{bp}$. It should be noticed, that three lines had the same alleles of sizes 632, 855 and 1088, but the one had the alleles of sizes 855 and $1145 \mathrm{bp}$.

In all inbred lines with C-type of CMS the alleles of expected size 398 bp were identified. On the other hand, in lines with normal cytoplasm two alleles were determined: 383 and $424 \mathrm{bp}$. The similar situation was watched in lines with T-type of CMS. In one inbred line the allele of expected size $440 \mathrm{bp}$ was identify; other three lines shown the presence of alleles of different size $-659 \mathrm{bp}$. It should be noticed, that this allele was characterized for all lines which proved nonsterile.

There is some scientific works which dedicate to research of mitochondrial genes, in particular, chimeric genes C-, S- and T-type cytoplasm. The presence of another sizes alleles, two or three alleles in inbred lines with normal cytoplasm can be connected with specificity of mitochondrial genome organization. On the other hand, these alleles can be presented in the nuclear genome because they were not identified in the lines with sterile cytoplasm.

Thus, the explanation of this phenomenon requires more detail investigation. Therefore, PCR based methods provide for breeding with a quick and simple tool for screening cytoplasm when mitochondrial DNA is used as the template. In any case, our results can be useful for identifying not only the major male sterile cytoplasm types, but the fertile genotypes. 